\title{
Induction of GLUT4 by inhibiting IFN- $\gamma$ : a winning move to halt type 2 diabetes?
}

\author{
Raffaella Mormile $^{1}$
}

Accepted: 21 December 2015 /Published online: 29 December 2015

(C) Springer-Verlag Berlin Heidelberg 2015

\section{Dear Editor:}

The fluidity of cell membranes has been described as a central link in the association of fatty acids (FAs) with susceptibility to type 2 diabetes (T2D). Decreased membrane fluidity has been connected with insulin resistance. Obesity, especially excess fat around the waist, is considered a primary cause of insulin resistance. It is well known that insulin resistance is the basis of the development of T2D. Adipose inflammation is described as a key component of the pathophysiology in obesity-related insulin resistance, T2D, and downstream complications. T2D has long been defined as a disease of inflammation. A bulk of data suggests that the pro-inflammatory cytokine interferon- $\gamma$ (IFN- $\gamma$ ) plays a major role in the regulation of the inflammatory response that accompanies the generation of insulin resistance, obesity, and T2D. IFN- $\gamma$ is regarded as one of the most significant independent predictors of metabolic syndrome. Adequate membrane fluidity is essential to cell function. The fluidity of cell membranes is strongly linked to its FA composition. The more saturated the fats are in the membrane, the more rigid the membrane. Conversely, unsaturated fatty acids (UFAs) with the kinks produced by their double bonds increase membrane fluidity. UFAs have been assessed to have profound effects on membrane fluidity that, in turn, influences a variety of cellular functions including the properties of the insulin receptor and insulin sensitivity as well as glucose transport across membranes. Although the specific mechanism by which a shift from unsaturated toward saturated fatty acids in cell membranes disrupts insulin sensitivity is not well established, it has been speculated that a decrease in

Raffaella Mormile

raffaellamormile@alice.it

1 Division of Pediatrics and Neonatology, Moscati Hospital, Via A. Gramsci, 3-81031 Aversa, Italy
GLUT4 expression in adipocytes may represent a possibility. It has been found that IFN- $\gamma$ markedly reduces expression of insulin signaling proteins including the GLUT4 glucose transporter. Interestingly, the GLUT4 expression is downregulated in adipose tissue in obesity. GLUT4 is a major mediator of glucose removal from the circulation and a key regulator of whole-body glucose homeostasis. GLUT4 is one of the 13 sugar protein transporters that catalyzes hexose transport across cell membranes. Among these sugar transporters, GLUT4 is highly expressed in adipose tissue. GLUT4 in adipose tissue is indispensable for normal global glucose homeostasis, while insulin receptors in this tissue appear much less critical. Depletion of GLUT4 in either adipose tissue or skeletal muscles has been recognized to cause insulin resistance and propensity toward diabetes. Remarkable, overexpression of GLUT4 in the adipose tissue of musclespecific GLUT4-deficient mice has been shown to overcome the glucose intolerance and diabetes. It has been observed that UFAs exert an anti-inflammatory action on adipose tissue and especially on mature adipocytes. Although there is strong evidence about the beneficial effect of certain UFAs in the adequate proportion in patients with T2D, there are no concluding data in this respect. UFAs have been recognized to influence insulin metabolism, positively improving considerably insulin action. Intake of diets rich in PUFAs, particularly n-3 and n-6, has been shown to modulate the inflammatory response modifying the evolution of T2D. All these contentions led us to hypothesize that adequate UFA membrane levels may counteract the generation of insulin resistance through the induction of GLUT4 expression as a result of inhibition of IFN- $\gamma$ production. Understanding the impact of the interaction between IFN- $\gamma$ and GLUT4 on the onset of T2D may provide new insights into the pathogenesis and prevention of this condition. Administration of anti-IFN- $\gamma$ may be a promising therapy to halt or prevent $\mathrm{T} 2 \mathrm{D}$, it being understood that diet and exercise are mandatory. 